\title{
Metodologías en la enseñanza del cálculo de probabilidades en undécimo grado, educación secundaria
}

\author{
Fernando Manuel Amador Núñez ${ }^{1}$ \\ Melvin Leonel Reyes Gómez ${ }^{2}$ \\ William Oswaldo Flores López ${ }^{3}$
}

\section{Resumen}

En este artículo se han analizado las metodologías en la enseñanza del cálculo de probabilidades en undécimo grado de educación secundaria. Se trata de un estudio cualitativo y cuantitativo con un diseño en el nivel descriptivo, donde participaron estudiantes y profesores de Matemática en Educación Secundaria. La información se recogió a partir de la observación, la guía de análisis de contenido, entrevista, cuestionario cognitivo y encuesta. El análisis se realizó con el software de estadística para la Ciencias Sociales, Statistical Product and Service Solutions (SPSS) y una matriz de lista de control. Los resultados muestran que las metodologías didácticas utilizadas en la enseñanza del cálculo de probabilidades se han caracterizado por un modelo de enseñanza de aprendo, practico y aplico, teniendo en cuenta situaciones-problemas de la vida cotidiana y del contexto de la población de estudio. No obstante, la falta de interés del estudiantado hacia la asignatura de Matemática tiene una influencia negativa hacia el cálculo de probabilidades.

Palabras clave: Metodologías; enseñanza; evaluación; aprendo-practico-aplico; situaciones de la vida cotidiana; interés hacia las matemáticas.

\section{Summary}

This article analyzed the teaching methodologies in calculus of probability in eleventh grade of secondary education. This is a qualitative and quantitative study with a design based on the descriptive level, where students and Mathematics teachers in Secondary Education participated. The information was collected through observation process, the guide of content of analysis, interviews, cognitive questionnaire and survey.

1 Licenciado en Ciencias de la Educación con mención en Matemáticas, Profesor de Matemática del Ministerio de Educación del Municipio de Nueva Guinea, feramador85@gmail.com

2 Licenciado en Ciencias de la Educación con mención en Matemáticas, Profesor de Matemática del Ministerio de Educación del Municipio de Nueva Guinea, melvin.gomez@gmail.com

3 Máster en Investigación en Didáctica de las Ciencias Experimentales y Matemática, Gestor de Proyectos de la Universidad de las Regiones Autónomas de la Costa Caribe Nicaragüense, william.flores@uraccan.edu.ni 
The analysis was performed using statistical software for Social Sciences, Statistical Product and Service Solutions (SPSS) and a matrix checklist. The results show that the teaching methods used in teaching calculus of probability have been characterized by a teaching model to learn, practice and apply, taking into account situations-problems of the daily life and context of the population under study. However, the lack of interest of students towards the subject of Mathematics has a negative influence to the calculus of probability.

Keywords: Methodologies; teaching; evaluation; learn-practice-apply; situations of daily life; interest towards Mathematics.

\section{Introducción}

La importancia de la Matemática en el cálculo de probabilidades en la vida cotidiana y en el currículo es visible en diversos documentos y publicaciones. Por ejemplo, en la última transformación curricular del Subsistema de Educación Básica y Media de Nicaragua se ha reconocido la necesidad de formar al estudiantado en el conocimiento y razonamiento del cálculo probabilístico, cuya finalidad se centra en que los estudiantes deben desarrollar habilidades, destrezas, aptitudes, actitudes y valores que propicie un pensamiento crítico, creativo, imaginativo, espacial y lógico, adaptarse al medio, actuar con autonomía y seguir aprendiendo para mejorar su calidad de vida.

Como consecuencia, el objetivo de este trabajo es analizar las metodologías didácticas en la enseñanza del cálculo de las probabilidades en undécimo grado de Educación Secundaria, y si estás metodologías influyen positivamente en el rendimiento académico de la asignatura de Matemática. El propósito es averiguar los métodos, técnicas y estrategias utilizadas en la enseñanza del cálculo de las probabilidades, y sí existe una relación entre los contenidos desarrollados con los procedimientos de evaluación.

\section{Revisión de literatura}

\section{Métodos de enseñanza de las probabilidades}

En la línea de métodos de enseñanza del cálculo de probabilidades sobresalen Tauber, Batanero y Sánchez (2005), los cuales diseñan una secuencia de aprendizaje basada en el uso de ordenadores, donde los estudiantes adquieren los diferentes elementos del significado de la "distribución normal", a partir de la resolución de problemas de análisis de datos. La tecnología introduce nuevos significados en los conceptos que se trata de enseñar, lo cual conlleva a nuevas formas de evaluación, basadas en trabajos abiertos y tareas complejas de análisis de datos. Una perspectiva similar es seguida por Alvarado y Batanero (2006) para el estudio del aprendizaje del "teorema central del límite". 
Igualmente se han analizado las concepciones espontáneas de los sujetos adultos sobre conceptos de estocástica avanzada (Ruiz, Albert \& Batanero, 2006), así como la resistencia de ciertas concepciones incorrectas y heurísticas tras un aprendizaje basado en el cambio conceptual (Sáenz, 1998; Barragués, 2002; Guisasola \& Barragués, 2002). Otras metodologías se centran en la evaluación de la capacidad de los estudiantes para resolver problemas de probabilidad, las variables que afectan la dificultad de las tareas y los posibles errores conflictos en el proceso de resolución (Cañizares, 1997; Lonjedo \& Huerta, 2005; Díaz, 2005).

\section{Significados de la probabilidad}

En Batanero (2005) y Batanero y Díaz (2007) se realiza un análisis sobre cuáles procedimientos ligados a los significados de la probabilidad son más relevantes para la enseñanza escolar. Estos significados se describen a continuación:

Significado intuitivo: Es muy adecuado en la educación primaria, pues el interés de los niños por los juegos puede usarse en la enseñanza para introducir la noción de probabilidad reconociendo la impredecibilidad de los resultados, los niños pueden percibir que algunos sucesos merecen más confianza que otros, en función de su experiencia (Batanero, 2005).

Significado clásico: Godino, Batanero y Cañizares (1987) indican que esta definición fue discutida desde su publicación, debido a debilidades tales como: es circular, utiliza el término que quiere definir (equiprobable), e introduce un elemento subjetivo asociado a la necesidad de juzgar la equiposibilidad de diferentes resultados. Por otro lado, esta definición no es aplicable cuando los experimentos tienen posibilidades infinitas (la variable es continua) o no se cumple la equiprobabilidad (el espacio muestral finito no es simétrico).

Significado frecuencial: Para Fernandes, Batanero, Contreras y Díaz (2009), el significado frecuencial es adecuado en la enseñanza, porque tiene una aplicación más amplia que el clásico en muchos fenómenos de la vida real y conecta la estadística con la probabilidad. Además, las posibilidades actuales de simulación facilitan el tratamiento de este enfoque.

Significado subjetivo: Desde el punto de vista de la enseñanza, Godino, Batanero y Cañizares (1987), sugieren usar en forma intuitiva este enfoque, en la educación primaria, con situaciones cotidianas del niño; se comenzaría asignando valores por parte del niño a las probabilidades, que se revisarían posteriormente con nuevas experiencias.

Significado axiomático: La enseñanza de la probabilidad, bajo esta axiomatización, sólo sería aconsejable a nivel universitario; cuando se hayan comprendido 
las definiciones clásica, frecuencial y subjetiva (Godino, Batanero y Cañizares,1987), aunque los alumnos de secundaria podrían comprenderla a nivel intuitivo.

\section{Materiales y métodos}

El enfoque de este estudio es cualitativo y cuantitativo. La integración del enfoque cualitativo permitió describir e interpretar la realidad educativa con el fin de llegar a la comprensión o a la transformación de dicha realidad, a partir del significado atribuido por las personas que la integran (Bisquerra, 2009). El método cuantitativo nos ayudó al tratamiento de los datos a través de la categorización y descripción de las propiedades, características y los perfiles de las personas, grupos, comunidades, procesos y objetos o cualquier otro fenómeno que se someta a un análisis (Hernández, Fernández, \& Baptista, 2010).

Mientras que su diseño es descriptivo, porque tuvo como objetivo indagar la incidencia de las modalidades o niveles de una o más variables en una población (Hernández, et al., 2010). Los participantes de estudios son estudiantes, del undécimo grado de Educación Secundaria y profesorado de Matemática del Colegio "Vigarny Flores Castle", situado en la Colonia Río Plata, Nueva Guinea, Región Autónoma de la Costa Caribe de Nicaragua. Los instrumentos utilizados fueron: guía de análisis de contenido, la entrevista, la observación, cuestionario cognitivo y encuesta. El análisis se realizó utilizando el software de estadística para la Ciencias Sociales (SPSS) y una matriz de lista de control.

\section{Resultados y discusión}

\section{Cálculo de probabilidades en undécimo grado}

El análisis de contenido realizado en el programa y libro de texto de matemática nos brinda que la unidad cálculo de probabilidades en undécimo grado se centraliza en la competencia de resolver problemas de probabilidad y probabilidad condicional, tipificándose en:

- Aplicarlos conceptos de probabilidades de complemento y la suma en situaciones prácticas.

- Calcular la probabilidad condicional de sucesos aleatorios relacionada con situaciones prácticas.

También promueve actividades donde se desarrollen los talentos, las habilidades y pensamientos creativos que contribuyan al alcance de logros personales y al fortalecimiento de la autoestima en el ámbito familiar, escolar y comunitario. En este sentido, las actividades de aprendizaje sugeridas, véase en el cuadro No. 1. 
Cuadro No. 1: Actividades de aprendizaje en la unidad de probabilidades

\begin{tabular}{|c|}
\hline Actividades de aprendizaje sugeridas en probabilidad \\
\hline $\begin{array}{l}\text { - Consulta el recurso educativo "Probabilidad y Juego" y video educativo "Probabilidad" para apoyarse en el desa- } \\
\text { rrollo del contenido. } \\
\text { - Mediante lluvia de ideas, respetando las ideas de los demás, habla lo que sabe acerca de las probabilidades, } \\
\text { cuáles son, en qué se caracterizan, a qué hacen referencia, cuál es su fórmula. } \\
\text { - Analiza a través de un ejemplo la probabilidad de un evento complementario: En el evento A (día nublado), } \\
\text { P(A)=0,3, la probabilidad de tener un día despejado será P(A`)=1- P(A)=0,7 } \\
\text { - Concluye que "La probabilidad del complemento de un evento A, son todos los elementos en un espacio } \\
\text { muestral (S) que no se encuentran en A. P(A') =1- P(A). } \\
\text { - Concluye con ayuda de los docentes que } 2 \text { o más eventos son independientes cuando la ocurrencia o no ocu- } \\
\text { rrencia de un evento no tiene efecto sobre la probabilidad de ocurrencia del otro evento (o eventos). Un caso } \\
\text { típico de eventos independientes es el muestreo con reposición, es decir, una vez tomada la muestra se regresa } \\
\text { de nuevo a la población donde se obtuvo. }\end{array}$ \\
\hline Actividades de aprendizaje sugeridas en probabilidad condicional \\
\hline $\begin{array}{l}\text { - Organizados en equipos practicando la equidad y el respeto a la diversidad atiende la orientación de los do- } \\
\text { centes al realizar el siguiente trabajo con tres urnas: la primera con } 3 \text { bolas blancas y } 2 \text { negras, la segunda con } 2 \\
\text { bolas blancas y } 4 \text { negras, y la tercera con } 2 \text { bolas blancas y } 6 \text { negras. } \\
\text { - Una o un estudiante pasa a extraer una bola de una urna elegida al azar y resulta ser blanca (Al hacer el experi- } \\
\text { mento la bola es blanca o negra, dependiendo del color de la bola que se saca, el docente cambiará el color, en } \\
\text { lugar de blanco poner negro). ¿Cuál es la probabilidad de que se haya extraído de la segunda urna? } \\
\text { - En caso de que los estudiantes tengan dificultades para resolver el problema, la o el docente planteará algunas } \\
\text { de las siguientes preguntas para orientarles: ¿Cuáles son los eventos que se tienen? ¿Qué probabilidad es la } \\
\text { que debemos calcular? ¿En la urna } 2 \text { cuál es la probabilidad de que sea bola blanca? ¿Cuál es el total de casos } \\
\text { de que sea bola blanca? ¿Cuáles son los casos favorables? } \\
\text { - Algunos estudiantes podrán resolverlos de distintas maneras utilizando técnicas de conteo, diagramas de árbol, } \\
\text { etc. } \\
\text { - Los equipos expondrán sus resultados y los docentes fomentarán la confrontación y guiarán a determinar la } \\
\text { probabilidad condicional. } \\
\text { - Expone con respeto y cordialidad en los diferentes equipos los resultados y con apoyo o guía de los docentes } \\
\text { se determinará la probabilidad condicional. } \\
\text { - Resuelve en equipo problemas que conlleve la utilización de probabilidad condicional. } \\
\text { Presenta los resultados en equipo manteniendo la cientificidad, el orden y la responsabilidad entre los integran- } \\
\text { - Elige algunos temas de la vida cotidiana y resuelve problemas utilizando las probabilidades. }\end{array}$ \\
\hline
\end{tabular}
Fuente: Elaboración propia.

El programa de Matemática de undécimo grado propone los siguientes criterios de evaluación en la unidad de probabilidades:

- Valora la aplicación de conceptos de probabilidades de complemento y de suma al realizar ejercicios de eventos relacionados con situaciones prácticas.

- Constata la participación, orden y respecto entre los estudiantes al realizar las diferentes actividades en la clase.

- Estimula y reconocer los diferentes talentos de los estudiantes en el aula de clases.

- Verifica la capacidad de los estudiantes al calcular la probabilidad condicional de sucesos aleatorios, relacionada con situaciones o eventos de la vida cotidiana. 


\section{EDUCACIÓN}

- Supervisa la práctica de valores de tolerancia respecto, solidaridad, responsabilidad y honestidad en la resolución de problemas empleando las probabilidades.

- Estimula el carácter investigativo y científico al realizar diferentes problemas y plantea, alternativas de solución empleando el cálculo de probabilidades.

\section{Metodologías en la enseñanza del cálculo de probabilidades en undécimo grado}

Las metodologías en la enseñanza de las probabilidades en undécimo grado, se puntualizan en el planeamiento didáctico de la evaluación y el modelo aprendo, practico y aplico, que se describe a continuación.

\section{- El planeamiento didáctico}

El planeamiento didáctico que el profesorado de Matemática realiza, es un proceso que permite seleccionar y organizar las variadas situaciones y experiencias de aprendizaje y evaluación que realizará el estudiantado de forma individual o en el grupo, con la mediación del docente, para obtener resultados óptimos en el desarrollo de las competencias e indicadores propuestos. Los resultados del análisis de la entrevista aplicada a los docentes de Matemática, permite que este se plantee interrogantes sobre la mejor manera en que pueden desarrollar su práctica pedagógica de forma efectiva, para ello parten de la reflexión sobre:

Cuadro No. 2: Interrogantes en el proceso de planificación

\begin{tabular}{|l|l|}
\hline \multicolumn{1}{|c|}{ Preguntas a reflexionar } & \multicolumn{1}{c|}{ Práctica pedagógica a implementar } \\
\hline ¿Qué está pasando? & Diagnóstico. \\
\hline ¿Qué se quiere hacer? & $\begin{array}{l}\text { Elaboración de actividades-acciones a realizar considerando el } \\
\text { apoyo al momento del proceso de enseñanza y aprendizaje y } \\
\text { su secuencia. }\end{array}$ \\
\hline $\begin{array}{l}\text { ¿Cómo se va a hacer? } \\
\text { a quiénes va dirigido? }\end{array}$ & $\begin{array}{l}\text { Actividades metodológicas para alcanzar competencias y los } \\
\text { indicadores de logros. }\end{array}$ \\
\hline ¿Con qué se va a hacer? & $\begin{array}{l}\text { Con grupos de docentes y estudiantes. } \\
\text { recursos tecnológicos, antologías didácticas. }\end{array}$ \\
\hline $\begin{array}{l}\text { ¿Cuánto tiempo se requiere } \\
\text { para hacerlo? }\end{array}$ & $\begin{array}{l}\text { Tiempo necesario para desarrollar su plan didáctica de acuer- } \\
\text { do a la programación pre-establecida. }\end{array}$ \\
\hline ¿Dónde se realizará? & Aula, patio, biblioteca, comunidad, otros. \\
\hline ¿Cómo se evaluara? & $\begin{array}{l}\text { La evaluación se realizará conforme a lo programado, es decir, } \\
\text { una evaluación de los aprendizajes del estudiantado. }\end{array}$ \\
\hline
\end{tabular}

Fuente: Elaboración propia. 
En este contexto, los profesores argumentaron que la estructura y los aspectos a incluir en el planeamiento didáctico son los indicadores de logro, contenidos básicos y estrategias metodológicas: Que se tipifican en aspectos como:

- Indicador de logro: aquí es importante incluir cuáles son los aprendizajes esperados que se propone desarrolle el estudiantado, una vez que concluya la clase.

- Contenidos básicos: estos se derivan de los indicadores de logro, ya que son un medio para alcanzar los aprendizajes esperados. Estos son el medio para desarrollar los indicadores de logro y competencias de grado.

- Estrategias metodológicas: acá se incluyen las situaciones de aprendizajes que desarrollará en el aula de clase, es decir todas las actividades vinculadas con los contenidos y con los ejes transversales del currículo, las estrategias metodológicas que promueven la motivación del estudiantado, de manera que ésta sea dinámica, atractiva y participativa.

En definitiva, el cambio que supone el enfoque de aprendizaje basado en competencias demanda que el docente adquiera un nuevo papel (Celik, 2011; Capraro, Capraro \& Helfeldt, 2010; Knight, 2008; Tigelaar, 2004). El profesor debe centrarse en lograr unos resultados de aprendizaje del estudiante, con el fin de que éste llegue a dominar el desempeño de una o varias competencias (Villa, Campo, Arranz, Villa \& García, 2013). Para ello, el profesor necesita planificar el desarrollo de estas competencias, a lo largo de la unidad programática. También debe manejar las metodologías y estrategias necesarias para desarrollar cada indicador de logro.

\section{- Planeamiento de la evaluación de los aprendizajes}

El planteamiento de la evaluación de los aprendizajes es el proceso por medio del cual se recolecta evidencia que permita establecer los logros de los estudiantes en cuanto a sus aprendizajes para poder emitir juicios de valor y tomar decisiones. En este contexto, los profesores explican que al momento de planificar la evaluación en el aula, es conveniente tomar en cuenta las siguientes fases:

Cuadro No. 3: Fases de planificación de la evaluación

\begin{tabular}{|l|l|}
\hline \multicolumn{1}{|c|}{ Fases de la evaluación } & \multicolumn{1}{c|}{ Claves para la ejecución de las fases de la evaluación } \\
\hline $\begin{array}{l}\text { Fase 1: La planificación de la } \\
\text { evaluación. }\end{array}$ & $\begin{array}{l}\text { ¿Qué evaluaré? ¿Para qué evaluaré? ¿Cómo evaluaré? ¿Con qué instrumen- } \\
\text { tos? }\end{array}$ \\
\hline $\begin{array}{l}\text { Fase 2: La recolección y selec- } \\
\text { ción de la información. }\end{array}$ & $\begin{array}{l}\text { La obtención de información sobre los aprendizajes del estudiantado, se } \\
\text { realiza mediante técnicas formales, semi-formales o no formales. Para que la } \\
\text { información sea más confiable y significativa se debe proceder realizando } \\
\text { aplicaciones sistemáticas de técnicas e instrumentos y no del simple azar. } \\
\text { Por otra parte, la información es significativa si se refiere a los aspectos rele- } \\
\text { vantes del aprendizaje. }\end{array}$ \\
\hline
\end{tabular}




\begin{tabular}{|l|l|}
\hline \multicolumn{1}{|c|}{ Fases de la evaluación } & \multicolumn{1}{c|}{ Claves para la ejecución de las fases de la evaluación } \\
\hline $\begin{array}{l}\text { Fase 3: Interpretación y valo- } \\
\text { ración de la información. }\end{array}$ & $\begin{array}{l}\text { Se realiza en términos del grado de desarrollo de los aprendizajes estableci- } \\
\text { dos. Se trata de encontrar sentido a los resultados de la evaluación, determi- } \\
\text { nar si son coherentes o no con los propósitos planteados y emitir un juicio } \\
\text { de valor. En la interpretación de los resultados también se considera las rea- } \\
\text { les posibilidades del estudiantado, sus ritmos de aprendizaje, la regularidad } \\
\text { demostrada, y otros. Porque ello determina el mayor o menor desarrollo de } \\
\text { las competencias y actitudes. }\end{array}$ \\
\hline Fase 4: Toma de decisiones. & $\begin{array}{l}\text { Los resultados de la evaluación deben llevarnos a aplicar medidas pertinen- } \\
\text { tes y oportunidades para mejorar el proceso de aprendizaje. Esto implica } \\
\text { volver sobre lo actuado para atender aquellos aspectos que requieran re- } \\
\text { adecuaciones, profundización, refuerzo o recuperación. Las deficiencias que } \\
\text { se produzcan pueden provenir tanto de las estrategias empleadas por el } \\
\text { docente como las previstas en el proceso de planificación. }\end{array}$ \\
\hline $\begin{array}{l}\text { Fase 5: Comunicación de los } \\
\text { resultados. }\end{array}$ & $\begin{array}{l}\text { Esto significa que se analiza y se dialoga acerca del proceso educativo con la } \\
\text { participación del estudiantado, profesorado y la familia, de tal manera que } \\
\text { los resultados de la evaluación son conocidos por todos los interesados. Así, } \\
\text { todos se involucran en el proceso y los resultados son más significativos. } \\
\text { Los instrumentos empleados para la comunicación de los resultados son los } \\
\text { registros auxiliares del profesorado y los boletines escolares. }\end{array}$ \\
\hline
\end{tabular}

Fuente: Elaboración propia.

En general, un proceso educativo basado en competencias, la evaluación de los estudiantes es un aspecto central, tanto en el proceso de aprendizaje como en los resultados del mismo (Ortega-Navas, 2010; Álvarez-Rojo, 2009; Cano, 2008; Perrenoud, 2004). Las técnicas y procedimientos para llevar a cabo un buen seguimiento de los estudiantes, dándoles realimentación en los momentos y con las actividades adecuadas, y elaborar pruebas sumativas que sean representativas de las competencias desarrolladas, es otro de los elementos que más debe trabajar el profesorado de hoy (DarlingHammond, et al., 2011). Distintas técnicas son las que promueve el profesorado, desde los portafolios (Dannefer \& Henson, 2007), la observación, el ensayo, el proyecto, las pruebas objetivas, las pruebas orales, la entrevista y pregunta (Navarrete et al., 2010), hasta las que pueden aportar hoy las nuevas tecnologías (Gikandi, Morrow, \& Davis, 2011). También se destaca la importancia que la autoevaluación y evaluación entre iguales en este enfoque de aprendizaje basado en competencias (Villa et al., 2013).

\section{- Aprendo, practico y aplico}

La observación aplicada en el aula de clases ayuda a describir el método de enseñanza del profesorado de Matemática, metodología que aporta a la práctica pedagógica en las aulas de clases, y caracterizan en tres momentos, ver cuadro No. 4. 
Cuadro No. 4: Proceso metodológico en el modelo aprendo, practico y aplico

\begin{tabular}{|l|l|}
\hline \multicolumn{1}{|c|}{ Modelo } & \multicolumn{1}{c|}{ Proceso Metodológico } \\
\hline Aprendo & $\begin{array}{l}\text { Tiene diversas intenciones, entre otras el de estrategias iniciales que sirven } \\
\text { de exploración de los saberes de los estudiantes en relación con el tema } \\
\text { (conocimiento previos). }\end{array}$ \\
\hline Practico & $\begin{array}{l}\text { Busca que el estudiante ejercite, practique, se apropie del nuevo aprendi- } \\
\text { zaje mediante diferentes actividades tales como: resolución de problemas. } \\
\text { También se presenta ejercicios de evaluación formativa a través de la autoe- } \\
\text { valuación y la co-evaluación. }\end{array}$ \\
\hline Aplico & $\begin{array}{l}\text { Busca que el estudiante aplique lo aprendido a situaciones reales, que recree } \\
\text { el aprendizaje, que saque provecho real a lo aprendido, que lo prepare para } \\
\text { su vida. }\end{array}$ \\
\hline
\end{tabular}

Fuente: Elaboración propia.

En suma, en el proceso de enseñanza-aprendizaje es un factor clave la metodología. Para un aprendizaje basado en competencias, además de los conocimientos, el docente debe crear situaciones y problemas en los que los estudiantes puedan desarrollar las competencias (Toraa-Bitlloch et al., 2012). Para lograrlo, el profesorado debe cambiar su rol hacia el acompañamiento y guía del estudiantado en su proceso de aprendizaje. Para ello, debe percibir al estudiantado como aprendiz activo y autorregulado, que obtenga a través de su propia experiencia el significado de su aprendizaje (Tigelaar, 2004). Según Hudson (2009). Los profesores competentes integran los distintos conocimientos sobre elementos como los contenidos, el currículo, la enseñanza-aprendizaje y los estudiantes. Asimismo, un estudio de Samuelowicz y Bain (2001) muestra que el profesorado que se orienta hacia una enseñanza centrada en el aprendizaje (con funciones como: previene confusión, ayuda al desarrollo de dominio, negocia, apoya la creación de conocimiento) logran en sus estudiantes unos resultados donde utilizan, organizan, transforman y personalizan los conocimientos.

\section{Dificultades asociadas a la enseñanza del cálculo de probabilidades en undécimo grado}

Los resultados de haber aplicado la observación, el cuestionario cognitivo y la encuesta en el estudiantado nos brinda información sobre las metodologías y dificultades asociadas a la enseñanza y aprendizaje del cálculo de probabilidades. Por ejemplo, el estudiantado argumenta que el profesorado de Matemática en la enseñanza de probabilidades usa una variedad de dinámicas. Lo que permite la motivación del estudiantado, por consiguiente, se interesen en aprender; sin embargo, según la observación se logró constatar que hace falta más explicación para que los estudiantes se apropien de los algoritmos a seguir para desarrollar los contenidos, además debe utilizarse metodologías de enseñanza, tales como: metodologías como la explicación formal de los contenidos, alumnos monitores, atención individualizada, trabajo en equipo. 


\section{EDUCACIÓN}

Con relativo al aprendizaje, se encontró que la edad de los estudiantes es un factor el cual interfiere en el aprendizaje, debido a la existencia del poco interés por aprender los contenidos matemáticos, además que no tiene el hábito de realizar las tareas, lo cual perjudica de manera directa en el aprendizaje, ya que no se retroalimenta la clase de manera individual, sabiendo que la manera más eficaz de aprender es la práctica. A esto, se le suma la problemática asociada al material didáctico para que el profesorado explique los contenidos al estudiantado y además emplee diversas estrategias de aprendizaje. Es indispensable que el estudiantado adquiera la bibliografía necesaria para que en la casa puedan estudiar los temas base que deben dominarse para el tema en estudio lo que resultó negativo al ser objeto de estudio, lo que determina que ellos no estudian en sus casas por cuenta propia porque no tienen materiales didácticos para hacerlo.

Otro aspecto relevante, es el interés de los estudiantes hacia la Matemática, los resultados brindan que el estudiantado goza de mucho interés en aprender esta materia, lo cual es un resultado objetivo en donde ellos creen que el tema en estudio les servirá mucho en su vida futura para resolver situaciones de su vida cotidiana, interés que no se relaciona con los resultados del cuestionario cognitivo que fue aplicado al estudiantado, se les asocia a un problema de comprensión de los conceptos de probabilidades, aunque el estudiantado consideran entenderle a las definiciones-conceptos de probabilidades, los resultados obtenidos en el cuestionario cognitivo fueron muy deficientes; ya que no fueron capaces de resolver ni la mitad de los ejercicios propuestos, lo que demuestra que estos estudiantes tienen muchas dificultades para resolver ejercicios de probabilidades y comprender los conceptos-definiciones, propiedades y características de las probabilidades.

\section{Conclusiones}

En cuanto al objetivo principal de esta investigación: analizar las metodologías didácticas en la enseñanza del cálculo de probabilidades en undécimo grado de Educación Secundaria, y sí influyen positivamente en el rendimiento académico de la asignatura de Matemática. Vemos que el profesorado de esta materia parte de las orientaciones curriculares que se transforman en planes didácticos con una estructura que se caracteriza por poseer indicadores de logros, contenido básico, estrategias metodológicas y una efectiva planificación de la evaluación de los aprendizajes. Con el propósito de emplear un modelo de enseñanza que se sustenta en tres fases: aprendo, aplico y practico.

Desde esta perspectiva, el profesorado de Matemática utiliza una variedad de dinámicas que orienta al estudiantado en los conceptos y definiciones del cálculo de probabilidades con situaciones de la vida cotidiana, lo que motiva el aprendizaje hacia este contenido. No obstante, los resultados muestran carencias de explicaciones concretas para que los estudiantes se apropien de los conceptos, propiedades y 
procedimientos que les permitirían realizar una buena argumentación en las situaciones-problemas que el profesorado les propone. Por otra parte, se encontró que tanto la edad del estudiantado y el interés hacia las matemáticas influye en la comprensión de los conceptos-definiciones de las probabilidades en undécimo grado. Estos dos factores, según Flores y Auzmendi (2015) se manifiestan por la valoración, el aprecio, la satisfacción, la curiosidad que los estudiantes sienten hacia la comprensión de los objetos matemáticos, es decir, el interés tanto por la disciplina como por su aprendizaje.

En general, las metodologías didácticas utilizadas en la enseñanza del cálculo de probabilidades se caracterizan por un modelo de enseñanza de aprendo, practico y aplico teniendo en cuenta situaciones-problemas de la vida cotidiana y del contexto de la población de estudio. Sin embargo, la falta de interés del estudiantado hacia la asignatura de Matemática tiene una influencia negativa hacia este contenido.

\section{Lista de referencias}

Alvarado, H., \& Batanero, C. (2006). Desingning a study process of the central limit theorem for engineers. In A. Rossman \& B. Chance (Eds.). Proceedings of the Seventh International Conference on Teaching Statistics. Salvador (Bahia), Brasil: International Associationfor Statistical Education.

Álvarez-Rojo, V. (2009). Perfiles y competencias docentes requeridas en el contexto actual de la educación universitaria. Revista española de orientación y psicpedagogía, $20(3), 270-283$.

Batanero, C. (2005). Significados de la probabilidad en la educación secundaria. RevistaLatinoamericana de MatemáticaEducativa, 8(3), 247-264.

Batanero, C., \&Díaz, C. (2007). Meaning and understanding of mathematics. Teh case of probability. In J. Van Bendegen\& K. Fraçois (Eds.). Philosophical dimmensions in mathematics education (107-127). New York: Springer.

Barragués, J. I. (2002). La enseñanza de la probabilidad en primer ciclo de universidad. Análisis de dificultades y propuesta alternativas de orientación constructivista. Tesis Doctoral: Universidad del País Vasco.

Bisquerra, R. (2009). Metodología de la Investigación Educativa. Madrid: La Muralla.

Borovcnik, M. (2012). Multiple perspectives on the concept of conditional probability. Avances de Investigación en Didáctica de la Matemática, 2, 5-27.

Cano, M. E. (2008). La evaluación por competencias en la educación superior. Profesorado. Revista Currículum y Formación de Profesorado, (3), 1-16. 


\section{EDUCACIÓN}

Cañizares, M. J. (1997). Influencia del razonamiento proporcional y combinatorio y de creencias subjetivas en las intuiciones probabilísticas primarias. Tesis Doctoral: Universidad de Granada.

Capraro, M. M., Capraro R. M., \&Helfeldt, J. (2010). Do differing types of field experiences make a difference in teacher candidates' perceived level of competence? (Report). Teacher Education Quarterly, 37(1), 131-154.

Celik, S. (2011). Characteristics and Competencies for Teacher Educators: Addressing the Need for Improved Professional Standards in Turkey. Australian Journal of Teacher Education, 36(4), 73-87.

Dannefer E. F., \& Henson, L. C., (2007). The portfolio approach to competency-based assessment at the Cleveland Clinic Lerner College of Medicine. Academic Medicine, 82(5), 493-502.

Darling-Hammond, L., Amrein-Beardsley, A., Heartel, E.H., \& Rothstein, J. (2011). Getting Teacher Evaluation Right: A Background Paper for Policy Makers. National Academy of Education \& American Educational Research Association (AERA).

Díaz, C. (2005). Conflictos semióticos en el cálculo de probabilidades a partir de tablas de doble entrada. Biaix, 24, 84-91.

Fernandes, J. A., Batanero, C., Contreras, J. M. \& Díaz, C. (2009). A simulaçāoem Probabilidades e Estatística: potencialidades e limitaçōes. Quadrante, XVIII, (1:2), 161-183.

Flores W. O., \& Auzmendi, E. (2015). Análisis de la estructura factorial de una escala de actitud hacia las matemáticas. Aula de Encuentros, 17(1), 45-77.

Gikandi, J. W., Morrow, D., \& Davis, N. E. (2011). Online formative assessment in higher education: A review of the literature. Computers \& Education, 57(4), 2333-2351.

Godino, J. D., Batanero C., \& Cañizares, M. J. (1987). Azar y probabilidad. Fundamentos didácticos y propuestas curriculares. Madrid: Síntesis.

Guisasola, J., \& Barragués, J. I. (2002). Heurísticas y sesgos de los estudiantes de primer ciclo de universidad en la resolución de problemas de probabilidad. Enseñanza de las Ciencias, 20(2), 285-302.

Hernández, R., Fernández, C., \& Baptista, M. (2010). Metodología de la Investigación. México: McGraw-Hill.

Hudson, P. (2009). How Can Preservice Teacher Be Measured against Advocated Professional Teaching Standards? The Australian journal of teacher education, 34(5), 65-73. 
Knight, P. T. (2008). El profesorado en Educación Superior. Formación para la excelencia. Madrid: Narcea Ediciones.

Lonjedo, M, A., \& Huerta, P. (2005). The nature of the quantities in a conditional probability problema. Its influence in the problema resolution, Proceedings of CERNE IV. Online: http://cerme4.crm.es/Papers\%2odefinitius/5/wg5litofpapers.

Navarrete, C., Pérez, H., López, R., Saborío, M., Jarquín, H., Zapata, I., \& Canelo, J. (2010). Manual de Planeamiento Didáctico y Evaluación de los Aprendizajes en Educación Secundaria. Managua: MINED.

Ortega-Navas, M. C. (2010). Competencias emergentes del docente ante las demandas del espacio europeo de educación superior. Revista Española de Educación Comparada, (16), 305-327.

Ruiz, B., Albert, A. \& Batanero, C. (2006). An exploratory study of students' difficulties with random variables In $A$. Rossman y B. Chance (Eds.). Proceedings of the Seventh International Conference on Teaching.Statistics. Salvador (Bahia),Brasil: International Association for Statistical Education.

Perrenoud, P. (2004). Diez nuevas competencias para enseñar: Invitación al viaje. Barcelona: Grao.

Sáenz, C. (1998). Teaching probability for conceptual change. Educational Studies in Mathematics, 35 (3). 233-254.

Samuelowicz, K., y Bain, J. D. (2001). Revisiting Academics' Beliefs about Teaching and Learning. Highereducation, 41(3), 299-325.

Tauber, L., Batanero, C., \& Sánchez, V. (2005). Diseño, implementación y análisis de enseñanza de la distribución normal en un curso universitario, EMA, 9, 2005, 2, 82-105.

Tigelaar, D. (2004). The development and validation of a framework for teaching competencies in higher education. Highereducation, 48(2), 253-268.

Torra-Bitlloch, I., De Corral, I., \& Pérez, M. J. (2012). Identificación, desarrollo y evaluación de competencias docentes en la aplicación de planes de formación dirigidos a profesorado universitario. REDU: Revista de Docencia Universitaria, 10(2), 21.

Villa, A., Campo, L., Arranz, S., Villa, O., \& García, A. (2013). Valoración del profesorado de magisterio sobre el aprendizaje basado en competencias implantado. Profesorado. Revista Currículum y Formación de Profesorado,17(3), 35-55. 